\title{
Photomodulation of Two-Dimensional Self-Assembly of Azobenzene-Hexa-peri-hexabenzocoronene-Azobenzene Triads
}

\author{
Ian Cheng-Yi Hou, ${ }^{\dagger}$ Valentin Diez-Cabanes, ${ }^{\dagger}$ Agostino Galanti, ${ }^{\S}$ Michal Valášek, ${ }^{\| \odot}$ Marcel Mayor, ${ }^{\|, \perp}$

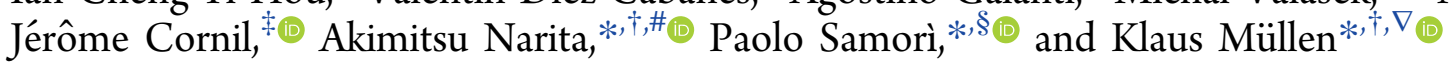 \\ ${ }^{\dagger}$ Max Planck Institute for Polymer Research, Ackermannweg 10, D-55128 Mainz, Germany \\ ${ }^{\ddagger}$ Laboratory for Chemistry of Novel Materials, University of Mons, Place du Parc 20, B-7000 Mons, Belgium \\ ${ }^{\S}$ Université de Strasbourg, CNRS, ISIS UMR 7006, 8 allée Gaspard Monge, 67000 Strasbourg, France \\ "Institute of Nanotechnology, Karlsruhe Institute of Technology KIT, P.O. Box 3640, 76021 Karlsruhe, Germany \\ ${ }^{\perp}$ Department of Chemistry, University of Basel, St. Johannsring 19, 4056 Basel, Switzerland \\ \#Organic and Carbon Nanomaterials Unit, Okinawa Institute of Science and Technology Graduate University 1919-1 Tancha, \\ Onna-son, Kunigami, Okinawa 904-0495, Japan \\ ${ }^{\nabla}$ Institute of Physical Chemistry, Johannes Gutenberg-University Mainz, Duesbergweg 10-14, D-55128 Mainz, Germany
}

Supporting Information

\begin{abstract}
Achieving exquisite control over self-assembly of functional polycyclic aromatic hydrocarbons (PAH) and nanographene (NG) is essential for their exploitation as active elements in (nano)technological applications. In the framework of our effort to leverage their functional complexity, we designed and synthesized two hexa-peri-hexabenzocoronene (HBC) triads, pAHA and oAHA, decorated with two lightresponsive azobenzene moieties at the pseudo-para and ortho positions, respectively. Their photoisomerization in solution is demonstrated by UV-vis absorption. ${ }^{1} \mathrm{H}$ NMR measurements of oAHA suggested $23 \%$ of $Z$-form can be obtained at a photostationary state with UV irradiation $(366 \mathrm{~nm})$. Scanning tunneling microscopy imaging revealed that the self-assembly of

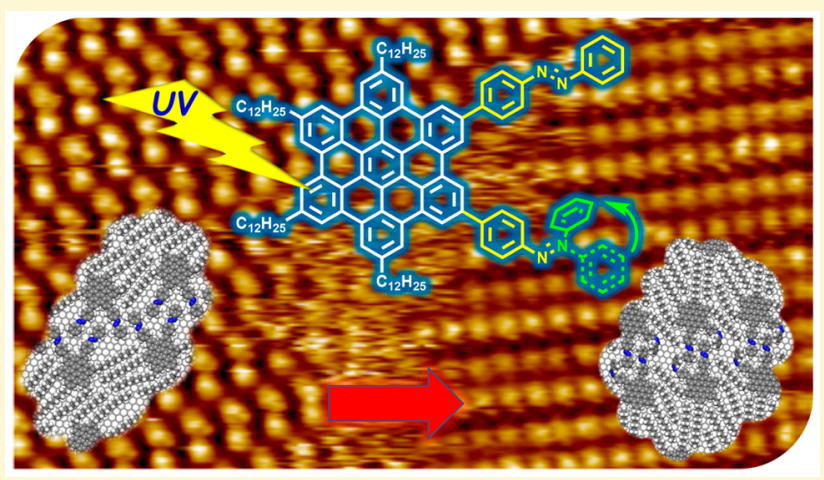
pAHA and oAHA at the solid-liquid interface between highly oriented pyrolytic graphite (HOPG) and their solution in 1,2,4trichlorobenzene can be modulated upon light irradiation. This is in contrast to our previous work using HBC bearing a single azobenzene moiety, which did not show such photomodulation of the self-assembled structure. Upon $E-Z$ isomerization both $p$ AHA and oAHA displayed an increased packing density on the surface of graphite. Moreover, $p$ AHA revealed a change of selfassembled pattern from an oblique unit cell to a dimer row rectangular crystal lattice whereas the assembly of oAHA retained a dimer row structure before and after light irradiation, yet with a modification of the inter-row molecular orientation. Molecular mechanics/molecular dynamics simulations validated the self-assembly patterns of $p$ AHA and $o$ AHA, comprising azobenzenes in their Z-forms. These results pave the way toward use of suitably functionalized large PAHs, as well as NGs, to develop photoswitchable devices.
\end{abstract}

\section{INTRODUCTION}

Hexa-peri-hexabenzocoronene (HBC), a molecule containing $42 \mathrm{sp}^{2}$ carbons and possessing a $D_{6 h}$ symmetry, is one of the most representative and well-studied polycyclic aromatic hydrocarbons (PAHs). With a diameter slightly exceeding 1 $\mathrm{nm}$, it is considered one of the smallest possible nanographenes (NGs), i.e., molecularly defined subunits of the graphene lattice which is confined in at least one of its lateral dimensions. $^{1-3}$ For this reason, HBC is frequently used as a model system to gain insight into the behavior of NG-based materials. $^{4-8}$

The large $\pi$-conjugated core of $\mathrm{HBC}$ gives rise to strong, noncovalent interactions among adjacent molecules, as well as between molecules and the basal plane of graphite. Understanding the resulting self-assembly processes is essential for advances in HBC-based (nano)technologies. ${ }^{5,6,9}$ Self-assembly of $\mathrm{HBC}$ derivatives has been studied in various environments, namely, in solution and in bulk, where $3 \mathrm{D}$ columnar supramolecular structures are formed, ${ }^{6,10-17}$ and on surfaces, enabling programmed 2D nanopatterning. ${ }^{14,18,19}$ Substituents at peripheral positions play a key role in controlling self-

Special Issue: Jean-Luc Bredas Festschrift

Received: April 17, 2019

Revised: June 12, 2019

Published: June 12, 2019 
assembly behavior of HBC. For example, HBCs with appropriate alkyl substituents show thermal phase transitions between crystalline and liquid-crystalline phases, where the phase-transition temperature can be tuned by the length of the side chains. ${ }^{6,11,20,21}$ Self-assembly of HBCs can thus be controlled by heat, yet the use of other external stimuli as remote controls to regulate self-assembly of $\mathrm{HBC}$-based materials has only been occasionally considered. ${ }^{19,22}$

Among external stimuli, light is particularly attractive, as photons may be remotely applied with high spatial and temporal resolution. A plausible way of imparting photoswitchable characteristics to $\mathrm{HBCs}$ is to functionalize their peripheries with photochromic moieties. ${ }^{23-26}$ In particular, azobenzene exhibits a pronounced geometric change between its $E / Z$-isomers, which has been applied to light-controllable, self-assembled materials and photomechanical devices. ${ }^{27-31}$ Photoresponsive self-assembly of azobenzene-containing materials has been intensively investigated, especially at solidair $^{32-36}$ and solid-liquid ${ }^{19,37-42}$ interfaces. Surface confinement restricts assembly in $2 \mathrm{D}$ and provides an opportunity for detailed examination of structures and dynamics of these molecules in real space by scanning probe microscopy techniques. In this context, it is extremely interesting to combine carbon-based nanostructures with photochromic molecules like azobenzene to develop optically responsive systems and materials. ${ }^{26} \mathrm{HBC}$ bearing one azobenzene unit was previously reported, but self-assembly of its $Z$-form could not be observed on a graphite surface by scanning tunneling microscopy (STM). ${ }^{19}$ Here we synthesized azobenzeneHBC-azobenzene triads, pAHA and oAHA (Scheme 1),

Scheme 1. Synthesis of Azobenzene-HBC-Azobenzene pAHA and oAHA

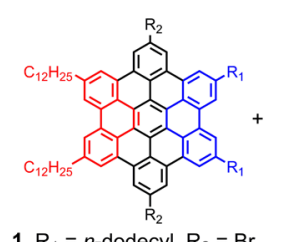

1, $\mathrm{R}_{1}=n$-dodecyl, $\mathrm{R}_{2}=\mathrm{Br}$
2, $\mathrm{R}_{2}=n$-dodecyl, $\mathrm{R}_{1}=\mathrm{Br}$

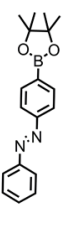

3

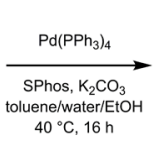

$40^{\circ} \mathrm{C}, 16 \mathrm{~h}$

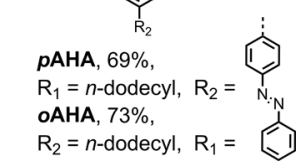

featuring an $\mathrm{HBC}$ core and two peripheral azobenzene moieties in the pseudo-para and pseudo-ortho positions, respectively. Geometries of $p$ AHA and oAHA are employed to explore intermolecular interactions that affect their selfassembly. Photoisomerization of the azobenzene moieties in solution is confirmed by UV-vis absorption, combined with ${ }^{1} \mathrm{H}$ NMR spectroscopy. Self-assembly of these two HBCs at the solid-liquid interface before and after UV light irradiation was monitored by STM and corroborated with molecular mechanics/molecular dynamics (MM/MD) simulations. Formation of stable self-assemblies for both pAHA and oAHA after isomerization of their azobenzene moieties reveals the potential of using large PAHs, as well as NGs, for photoresponsive devices and smart surfaces.

\section{RESULTS AND DISCUSSION}

Synthesis of Azobenzene-HBC-Azobenzene Triads. pAHA and oAHA were synthesized from the corresponding HBCs 1 and 2 bearing two bromo groups at desired positions, in addition to four $n$-dodecyl chains, ${ }^{43}$ which guarantee the molecule's good solubility in organic solvents and high affinity to graphite surfaces (Scheme 1). The azobenzene moieties were introduced by a 2 -fold Suzuki coupling reaction between 1 or 2 with azobenzene boronic ester $3 .^{44}$ pAHA and oAHA were isolated in $69 \%$ and $73 \%$ yields, respectively.

Photoisomerization in Solution. Reversible photoisomerization of both pAHA and oAHA could be observed in solution upon alternating irradiation at 366 and $436 \mathrm{~nm}$ (Figures S1-S3). The spectral changes upon photoisomerization were relatively small and could be attributed to overlapping absorption features of the azobenzene units and the HBC core. HBC has a higher extinction coefficient, ${ }^{15,45}$ which could overshadow spectral variation of the azobenzene units. Nevertheless, oAHA showed sufficient solubility to enable ${ }^{1} \mathrm{H}$ NMR spectroscopic analysis at room temperature, which demonstrated that approximately $23 \%$ of $Z$-form was obtained in a photostationary state (PSS) after irradiation at $366 \mathrm{~nm}$ (Figure S4).

STM Investigation of 2D Self-Assembly. The UV-vis absorption and ${ }^{1} \mathrm{H}$ NMR results, indicating the efficient photoisomerization of $p$ AHA and $o$ AHA in solution, motivated us to investigate the influence of isomerization on their selfassembly at the solid-liquid interface between graphite and their supernatant solutions. 2D self-assembly patterns of $p$ AHA and $o$ AHA were monitored by STM at the interface between 1,2,4-trichlorobenzene (TCB) solutions and highly oriented pyrolytic graphite (HOPG) before and after in situ UV light irradiation (Figures 1 and 2; Table 1). In all cases, brighter areas with a diameter of $\sim 1 \mathrm{~nm}$ can be assigned to $\mathrm{HBC}$ cores as a result of more favorable resonant tunneling between the Fermi level of HOPG and the frontier orbitals of the large aromatic core. Nonirradiated pAHA exhibits an oblique unit cell containing one molecule and an area of $6.8 \pm 0.6 \mathrm{~nm}^{2}$ per molecule (Figure 1a and Table 1), while oAHA forms a dimerrow structure with two molecules per unit cell and an area of $5.9 \pm 0.3 \mathrm{~nm}^{2}$ per molecule (Figure $2 \mathrm{a}$ and Table 1). A different assembly was also observed for oAHA (Figure S5; Table S1). These crystal packings are all assigned to assemblies of E,E-isomers, which have both azobenzenes in $E$-form (Figures 1a, 2a, and S5).

It is interesting to note that, although $p$ AHA and oAHA are a pair of isomers themselves, their assemblies show significantly different density where that of oAHA is much more compact. Different packing motifs between $p$ AHA and oAHA demonstrate that connectivity of the azobenzene-HBC-azobenzene triads strongly affects molecular self-assembly at the solidliquid interface. The same supramolecular packing is extended over a larger area $(50 \mathrm{~nm} \times 50 \mathrm{~nm})$ (Figures 1a and 2a, insets). Analysis of pAHA over even larger areas demonstrated that the crystalline domains end when terraces in the HOPG surface were encountered. These domains remained intact for hours without transforming into other self-assembled structures, thus confirming high stability of the crystal packing.

STM Investigation after Light Irradiation. After the 2Dself-assembly patterns of $p$ AHA and $o$ AHA were illustrated, their solution on the HOPG substrate was irradiated in situ with UV light $\left(\lambda_{\text {irr }}=366 \mathrm{~nm}\right)$ to examine the effect of photoisomerization of the azobenzene moieties on the $2 \mathrm{D}$ crystalline assemblies. After $3 \mathrm{~min}$ of irradiation at a power density of $2 \mathrm{~mW} / \mathrm{cm}^{2}$, new domains with radically different lattice parameters were observed for both pAHA and oAHA, along with the simultaneous presence of the aforementioned 
(a)

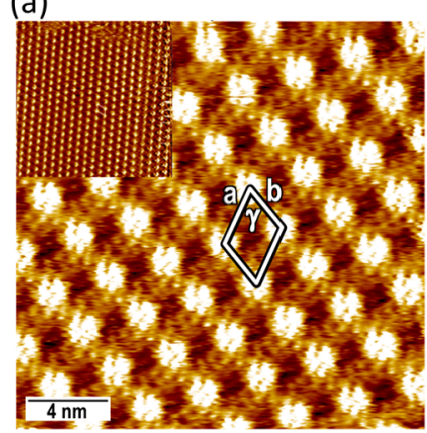

(c)

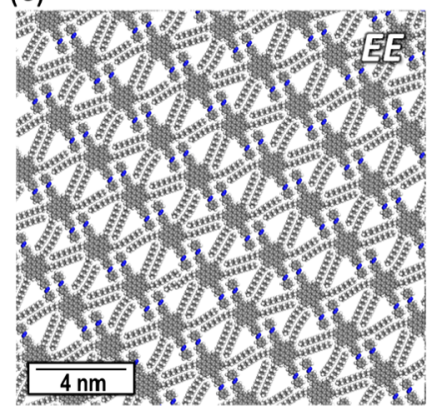

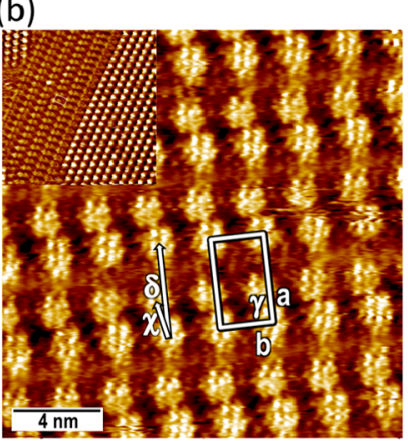

(d)

(b)

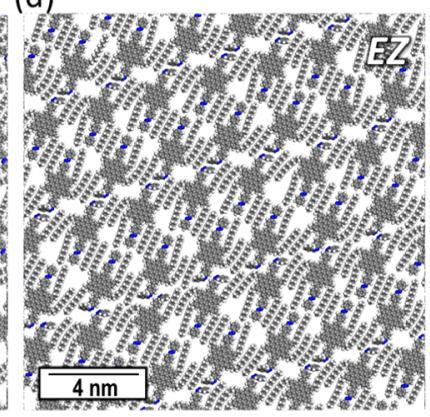

Figure 1. STM images recorded at the interface between HOPG and a $0.1 \mathrm{mM}$ solution of pAHA in TCB and their $2 \mathrm{D}$ crystal packing models derived from MM/MD simulations. (a) An STM image taken without irradiation (pAHA ori in Table 1) and (c) a corresponding packing model of $E, E$-isomers. (b) An image recorded after irradiation at $366 \mathrm{~nm}$ (pAHA irr in Table 1) and (d) a corresponding packing model of E,Z-isomers. Inset in $(\mathrm{a}, \mathrm{b})$ : images of the same crystal packing taken for a larger area of $50 \mathrm{~nm} \times 50 \mathrm{~nm}$. The newly formed dimer row crystal packing coexists with the oblique packing of $E, E$ isomers with a clear border, as can be seen in the inset of (b). Tunneling parameters: (a) bias voltage tunneling $\left(V_{\mathrm{T}}\right)=-300 \mathrm{mV}$, average tunneling current $\left(I_{\mathrm{T}}\right)=20 \mathrm{pA} ;(\mathrm{c}) V_{\mathrm{T}}=-300 \mathrm{mV}, I_{\mathrm{T}}=20$ pA. Atom color coding: (c, d) carbon (gray), nitrogen (blue), hydrogen (white).

domains formed by their E,E-isomers (Figures $1 \mathrm{~b}$ and $2 \mathrm{~b}$, respectively). Molecules in the photomodified domains assemble in dimer rows with a rectangular crystal lattice for both pAHA and oAHA showing an area of $5.3 \pm 0.2 \mathrm{~nm}^{2}$ and $4.6 \pm 0.3 \mathrm{~nm}^{2}$ per molecule, respectively (Figures $1 \mathrm{~b}$ and $2 \mathrm{~b}$ and Table 1). Additionally, another dimer row structured selfassembly pattern with $\delta$ close to $0^{\circ}$ and a parallelogram unit cell was observed for pAHA (Figure S6 and Table S1). HBC cores in the photomodified domains appear darker, compared with those in intact domains of the E,E-isomers, coexisting on the same STM image (inset of Figures $1 b$ and $2 b$ ). This could indicate a larger HOPG/HBC distance ${ }^{46}$ possibly induced by nonplanar $Z$-form azobenzene arms. Moreover, the photomodified domains exhibit denser packing than those in the original domains of the E,E-isomers, as evidenced by their smaller average area per molecule (Table 1). These observations support an assumption that the photomodified domains consist of molecules containing nonplanar $Z$-form isomers, with azobenzene arms presumably back-folded in the supernatant solution, reducing the molecular footprint visualized by STM. However, although rarely observed on HOPG for HBC species, ${ }^{10,47,48}$ occurrence of a change in a $2 \mathrm{D}$-assembly pattern for the $E, E$-isomers themselves cannot be completely excluded.

(a)

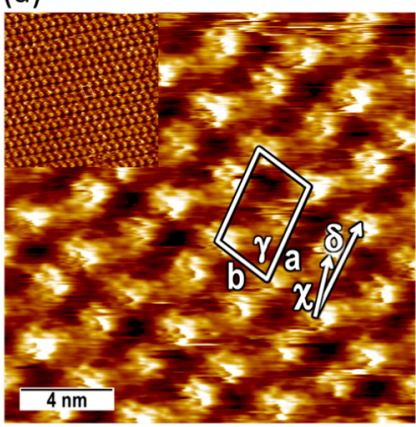

(c)

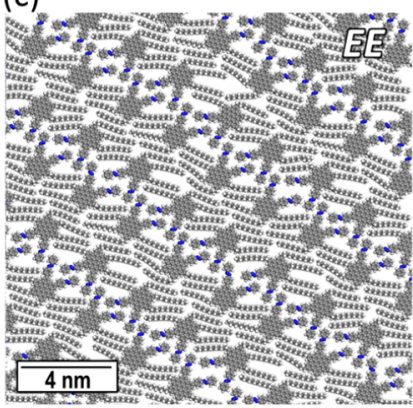

(b)

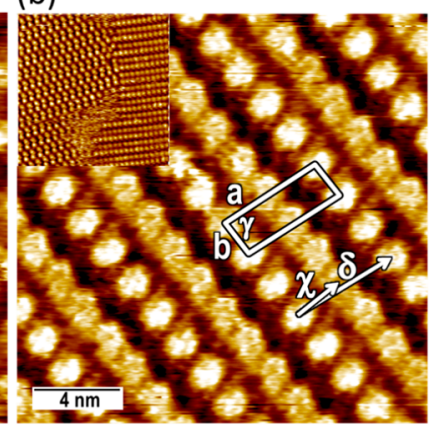

(d)

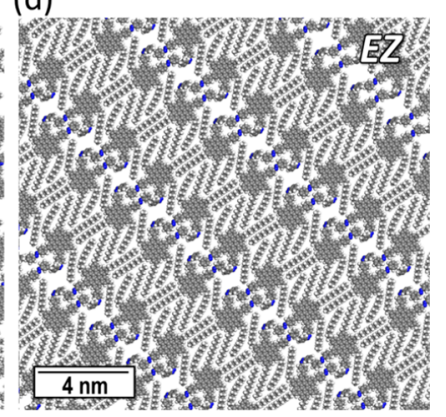

Figure 2. STM images recorded at the interface of HOPG and a 0.1 $\mathrm{mM}$ TCB solution of oAHA and their 2D crystal packing models derived from $\mathrm{MM} / \mathrm{MD}$ simulations. (a) An STM image taken without irradiation (oAHA ori in Table 1 ) and (c) a corresponding packing model of E,E-isomers. (b) An image taken after irradiation at $366 \mathrm{~nm}$ (oAHA irr in Table 1) and (d) a corresponding packing model of E,Zisomers. Inset in $(\mathrm{a}, \mathrm{b})$ : images of the same crystal packing taken over a larger area $50 \mathrm{~nm} \times 50 \mathrm{~nm}$. The newly formed rectangular dimer row crystal packing coexists with the domain of E,E-isomers with a clear border, as can be seen in the inset of (b). Tunneling parameters: (a) $V_{\mathrm{T}}=-550 \mathrm{mV}, I_{\mathrm{T}}=20 \mathrm{pA}$; (c) $V_{\mathrm{T}}=-700 \mathrm{mV}, I_{\mathrm{T}}=20 \mathrm{pA}$. Atom color coding: (c,d) carbon (gray), nitrogen (blue), hydrogen (white).

Simulation of the Assembly Patterns. MM/MD calculations were performed to further support the assumed formation of self-assembled domains consisting of isomers with $Z$-form azobenzenes, as well as to gain a greater understanding of how the azobenzene moieties govern the self-assembly of pAHA and oAHA by unraveling the packing at the atomistic level. Although lattice constants extracted from simulated results are generally larger than those experimentally determined, there is a reasonable agreement between experiment and simulation, allowing for a detailed interpretation of the STM images (Figure S7 and Table S2). Considering the relatively low $Z / E$-ratio at the PSS suggested by ${ }^{1} \mathrm{H}$ NMR measurements for oAHA (Figure S4) and assuming similar behavior for $p$ AHA, we infer that the assembly patterns arising after irradiation in both cases are composed of $E, Z$-isomers, but not Z,Z-isomers. The best matching simulated patterns of $E, Z$ isomers are displayed in Figures 1c,d and 2c,d. Interestingly, nanophase separations between flexible alkyl chains and rigid azobenzenes are suggested for the packing of E,E-isomers of both $p$ AHA and oAHA (Figures $1 \mathrm{c}$ and 2c, respectively). The nanophase separation disappears in the self-assembly of the E,Z-isomer of pAHA (Figure 1d). In the case of oAHA, azobenzenes intercalate in a zigzag fashion. Spatial demand of azobenzenes on the surface determines the length $b$ of the short lattice axis (Figure 2c): when one of the azobenzenes is switched into the $Z$-form, molecular size is reduced and the packing becomes tighter (Figure $2 \mathrm{~d}$ ). In contrast to $\mathrm{pAHA}$, the 
Table 1. Experimental Lattice Constants of $p$ AHA and $o$ AHA Self-Assembly at the HOPG/TCB Interface

\begin{tabular}{|c|c|c|c|c|c|c|c|c|}
\hline & $a(\mathrm{~nm})$ & $b(\mathrm{~nm})$ & $\gamma(\operatorname{deg})$ & $\chi^{b}(\mathrm{~nm})$ & $\delta^{b}(\mathrm{deg})$ & $\operatorname{area}^{c}\left(\mathrm{~nm}^{2}\right)$ & $E_{\mathrm{ads}}{ }^{d}(\mathrm{kcal} / \mathrm{mol})$ & $\mathrm{BE}^{e}(\mathrm{kcal} / \mathrm{mol})$ \\
\hline pAHA ori & $3.0 \pm 0.2$ & $2.5 \pm 0.1$ & $65 \pm 5$ & - & - & $6.8 \pm 0.6$ & $-210.89^{f}$ & $-23.35^{f}$ \\
\hline pAHA irr $^{a}$ & $4.2 \pm 0.1$ & $2.52 \pm 0.05$ & $88 \pm 1$ & $1.6 \pm 0.1$ & $11 \pm 3$ & $5.3 \pm 0.2$ & $-204.57^{g}$ & $-13.83^{g}$ \\
\hline oAHA ori & $4.4 \pm 0.1$ & $2.7 \pm 0.1$ & $81 \pm 3$ & $2.0 \pm 0.2$ & $25 \pm 4$ & $5.9 \pm 0.3$ & $-211.58^{f}$ & $-17.27^{f}$ \\
\hline oAHA $i r r^{a}$ & $5.2 \pm 0.2$ & $1.77 \pm 0.09$ & $83 \pm 5$ & $2.4 \pm 0.3$ & $-4 \pm 4$ & $4.6 \pm 0.3$ & $-205.72^{g}$ & $-19.50^{g}$ \\
\hline
\end{tabular}

${ }^{a}$ Only formed after irradiation at $366 \mathrm{~nm} .{ }^{b}$ Inter-row distance and angle, specified in STM figures. ${ }^{c}$ Area per molecule. ${ }^{d}$ Adsorption energy between molecule and the HOPG surface. Derived from MM/MD simulation. ${ }^{e}$ Intermolecular binding energy. Derived from MM/MD simulation. ${ }^{f}$ Calculated for the $E, E$-form. ${ }^{g}$ Calculated for the E,Z-form.

nanophase separation in $o$ AHA is retained in the self-assembly of both E,Z- and Z,Z-isomers (Figure S7). The incremental increase of binding energy with the number of $Z$-units in the oAHA molecule indicates that interaction between $Z$ azobenzenes benefits retention of the nanophase separation patterns (Table S2).

It is important to stress that observation of stable selfassemblies of molecules containing $Z$-isomers of azobenzenebased photoswitches at the solid-liquid interface by STM represents a challenging task. ${ }^{37,40}$ This is a result of the nonplanarity of the $Z$-isomers and lone-pair electrons of nitrogens interacting with the graphite surfaces, thereby causing molecular desorption. Formation of stable assemblies for different photoactive states without desorption/readsorption of molecules is essential for development of responsive surfaces and devices. ${ }^{45,49,50}$ In our case, the packing density increased upon irradiation with UV light, and we did not monitor the presence of regions with fuzzy contrast between domains of different assemblies. It is therefore likely that the physisorbed molecules undergo a process that involves the subsequential desorption, isomerization in solution, and readsorption onto the surface where void space is created by the shrinking of the assemblies. At the same time, some other molecules may undergo isomerization directly on the surface. Moreover, large PAHs such as HBC, namely, NGs, exhibit very high adsorption energy toward HOPG surfaces. ${ }^{51}$ In this regard, desorption of molecules from the surface might not occur while molecules may move on the surface during irradiation and rearrange to form the new domains. However, the absence of a monitoring of the switch on the time scale of the event does not allow us to provide a conclusive mechanism for this process. Note also from the MM/MD calculation that absolute values of adsorption energies for the different isomers of pAHA and oAHA are similar ( $<9 \%$ energy difference from the most to the least stable isomers, see Table S2). Such a scenario is consistent with our observation of self-assembly of the $Z$-azobenzene-containing isomers for both pAHA and oAHA.

\section{CONCLUSION}

In summary, we have synthesized two model compounds, pAHA and oAHA, to cast light onto the photoresponsive behavior of large PAHs, as well as NGs, decorated with azobenzenes. UV-vis absorption and ${ }^{1} \mathrm{H}$ NMR measurements demonstrated their photoisomerization in solution. STM images and MM/MD simulation of $p$ AHA and $o$ AHA revealed the photomodulation of self-assembled structures into more compact packings, formed by $Z$-azobenzene-containing isomers at the TCB/HOPG interface. The MM/MD simulation suggests similar nanophase separation between alkyl chains and azobenzenes in all three isomers of oAHA while it disappears in the E,Z-isomer of pAHA. The markedly different packings of the different isomers of $p$ AHA and $o$ AHA on the graphite surface provide clear evidence for the potential of using large PAHs, as well as NGs, for the design of new photoresponsive materials that are suitable for the development of switchable surfaces and devices. We are actively working toward gaining a complete understanding on the mechanism of this photomodulated self-assembly and exploring $\mathrm{HBC}$ derivatives possessing more azobenzene substituents and higher symmetry.

\section{EXPERIMENTAL METHODS}

General Details. All reactions working with air- or moisturesensitive compounds were carried out under argon atmosphere using standard Schlenk line techniques. Thin layer chromatography (TLC) was performed on silica gel coated aluminum sheets with F254 indicator. Silica gel column chromatography separation was performed with $0.063-0.200 \mathrm{~mm}$ particle size. Nuclear magnetic resonance (NMR) spectra were recorded in deuterated solvents using Bruker AVANCE III 500 and Bruker AVANCE III $700 \mathrm{MHz}$ NMR spectrometers. The ${ }^{13} \mathrm{C}$ NMR spectra were recorded with a spinecho attached-proton test (APT) sequence with $\mathrm{CH}, \mathrm{CH}_{3}$ showing negative signal and $\mathrm{C}, \mathrm{CH}_{2}$ showing positive signal. Chemical shifts $(\delta)$ were expressed in ppm relative to the residual of solvent $\left(\mathrm{C}_{2} \mathrm{D}_{2} \mathrm{Cl}_{4}\right.$ (a $6.00 \mathrm{ppm}$ for ${ }^{1} \mathrm{H}$ NMR and $73.78 \mathrm{ppm}$ for ${ }^{13} \mathrm{C}$ NMR). Coupling constants $(J)$ were recorded in Hertz $(\mathrm{Hz})$ with multiplicities explained by the following abbreviations: $\mathrm{s}=$ singlet, $\mathrm{d}=$ doublet, $\mathrm{t}$ $=$ triplet, $\mathrm{q}=$ quartet, $\mathrm{dd}=$ doublet of doublets, $\mathrm{dt}=$ doublet of triplets, $\mathrm{m}=$ multiplet, and $\mathrm{br}=$ broad. The UV-vis absorption spectra were measured with a PerkinElmer Lambda 900 spectrophotometer in a quartz cuvette (Hellma) with a light path of $1 \mathrm{~cm}$ at room temperature. High-resolution mass spectra (HRMS) were recorded by matrix-assisted laser decomposition/ionization (MALDI) using 7,7,8,8-tetracyanoquinodimethane (TCNQ) as matrix with a Bruker Reflex II-TOF spectrometer (MALDI-TOF HRMS).

Materials. Unless otherwise noted, all starting materials and reagents were purchased from commercial sources (Alfa Aesar, SigmaAldrich, Acros, and TCI) and used without further purification. HBCs 1 and 2 containing two bromo functional groups ${ }^{43}$ and the azobenzene boronic ester $3^{44}$ were synthesized according to the previously published literature procedures.

Synthesis of pAHA. To a suspension of dibromo-HBC 1 (40 mg, $0.030 \mathrm{mmol})$, SPhos $(4.4 \mathrm{mg}, 0.011 \mathrm{mmol})$, and azobenzene boronic ester $3(26 \mathrm{mg}, 0.084 \mathrm{mmol})$ in toluene $(8 \mathrm{~mL})$ was added a solution of $\mathrm{K}_{2} \mathrm{CO}_{3}(1.4 \mathrm{~g}, 10 \mathrm{mmol})$ in water $(1.5 \mathrm{~mL})$ and ethanol $(1.5 \mathrm{~mL})$. This mixture was degassed by the freeze-pump-thaw technique (1 cycle). $\mathrm{Pd}\left(\mathrm{PPh}_{3}\right)_{4}(8.0 \mathrm{mg}, 0.0069 \mathrm{mmol})$ was then added to the mixture, which was further degassed by the freeze-pump-thaw technique for another 2 cycles. The mixture was then heated at $40{ }^{\circ} \mathrm{C}$ under vigorous stirring for $16 \mathrm{~h}$. After cooling to room temperature, the mixture was poured into methanol. The yellow precipitate was collected by vacuum filtration and washed with methanol and then purified by silica gel column chromatography (two times, eluent: hot toluene) to afford the title compound as a bright yellow solid ( $32 \mathrm{mg}$, 69\%). ${ }^{1} \mathrm{H}$ NMR (500 MHz, $\left.\mathrm{C}_{2} \mathrm{D}_{2} \mathrm{Cl}_{4}, 373 \mathrm{~K}\right): \delta 8.60-8.40(\mathrm{br}, 4 \mathrm{H})$, $8.35-8.20(\mathrm{~m}, 4 \mathrm{H}), 8.20-8.05(\mathrm{~m}, 8 \mathrm{H}), 8.05-7.95(\mathrm{~m}, 8 \mathrm{H}), 7.75-$ $7.55(\mathrm{~m}, 6 \mathrm{H}), 3.15-2.85(\mathrm{br}, 8 \mathrm{H}), 2.15-1.90(\mathrm{br}, 8 \mathrm{H}), 1.85-1.60$ (br, $16 \mathrm{H}), 1.60-1.20(\mathrm{~m}, 56 \mathrm{H}), 1.10-0.85(\mathrm{br}, 12 \mathrm{H}) .{ }^{13} \mathrm{C}$ NMR 
$\left(126 \mathrm{MHz}, \mathrm{C}_{2} \mathrm{D}_{2} \mathrm{Cl}_{4}, 373 \mathrm{~K}\right): \delta 153.11,151.95,144.02,139.17$, $135.56,130.54,129.28,128.86,128.59,127.72,123.47,122.90$, 122.08, 121.99, 120.70, 120.66, 120.39, 118.67, 118.50, 118.42, 36.83, $31.70,31.58,29.99,29.78,29.71,29.67,29.62,29.49,29.12,22.39$, 13.74. HRMS (MALDI-TOF) Mass. Calcd. for $\mathrm{C}_{114} \mathrm{H}_{130} \mathrm{~N}_{4}[\mathrm{M}]^{+}$ 1555.0296. Found $[\mathrm{M}]^{+} 1555.0505$.

Synthesis of oAHA. To a suspension of dibromo-HBC $2(45 \mathrm{mg}$, $0.034 \mathrm{mmol})$, SPhos $(5.4 \mathrm{mg}, 0.014 \mathrm{mmol})$, and azobenzene boronic ester $3(41 \mathrm{mg}, 0.13 \mathrm{mmol})$ in toluene $(6 \mathrm{~mL})$ was added a solution of $\mathrm{K}_{2} \mathrm{CO}_{3}(0.7 \mathrm{~g}, 5 \mathrm{mmol})$ in water $(1 \mathrm{~mL})$ and ethanol $(1 \mathrm{~mL})$. This mixture was degassed by the freeze-pump-thaw technique (1 cycle). $\mathrm{Pd}\left(\mathrm{PPh}_{3}\right)_{4}(7.6 \mathrm{mg}, 0.0066 \mathrm{mmol})$ was then added to the mixture, which was further degassed by the freeze-pump-thaw technique for another 2 cycles. The mixture was then heated at $40{ }^{\circ} \mathrm{C}$ under vigorous stirring for $16 \mathrm{~h}$. After cooling to a room temperature, the mixture was poured into methanol. The yellow precipitates were collected by vacuum filtration and washed with methanol and then purified by silica gel column chromatography (two times, eluent: hot toluene) to afford the title compound as a brownish solid $(38 \mathrm{mg}$, $73 \%) .{ }^{1} \mathrm{H}$ NMR (500 MHz, $\left.\mathrm{C}_{2} \mathrm{D}_{2} \mathrm{Cl}_{4}, 373 \mathrm{~K}\right): \delta 8.20-8.05(\mathrm{~m}, 8 \mathrm{H})$, 8.00-7.87 (br, 2H), 7.85-7.80 (br, 2H), 7.80-7.76 (br, 2H), 7.76$7.71(\mathrm{br}, 2 \mathrm{H}), 7.71-7.60(\mathrm{~m}, 12 \mathrm{H}), 7.58-7.50(\mathrm{br}, 2 \mathrm{H}), 3.00-2.80$ (br, 4H), 2.80-2.65 (br, 4H), 2.05-1.93 (br, 4H), 1.93-1.78 (br, $4 \mathrm{H}), 1.78-1.20(\mathrm{~m}, 72 \mathrm{H}), 1.10-0.90(\mathrm{~m}, 12 \mathrm{H}) .{ }^{13} \mathrm{C}$ NMR $(126$ $\left.\mathrm{MHz}, \mathrm{C}_{2} \mathrm{D}_{2} \mathrm{Cl}_{4}, 373 \mathrm{~K}\right): \delta 153.15,151.72,143.32,138.73,138.51$, $134.22,130.48,128.85,128.61,128.55,128.27,128.20,128.01$, $127.91,127.19,123.24,122.90,122.65,121.68,121.48,120.27$, 120.20, 119.85, 118.09, 118.00, 117.80, 117.62, 117.10, 36.89, 36.66, $31.74,31.63,31.35,30.11,30.09,29.84,29.74,29.70,29.68,29.61$, 29.54, 29.42, 29.17, 22.43, 13.78. HRMS (MALDI-TOF) Mass. Calcd. for $\mathrm{C}_{114} \mathrm{H}_{130} \mathrm{~N}_{4}[\mathrm{M}]^{+}$1555.0296. Found $[\mathrm{M}]^{+} 1555.0420$.

Photoswitching in Solution. The photoswitching in solution was conducted by direct irradiation of a THF solution of pAHA or oAHA in a quartz cuvette used for UV-vis absorption spectral measurement. The absorption spectra were directly recorded using a UV-vis spectrophotometer after irradiation. The light source used for switching was a Mercury arc lamp (HBO 200W/2, OSRAM). The irradiation wavelength was controlled by using optical filters (Schott Glaswerke). More details of experimets can be found in the Supporting Information.

STM Investigation. The STM imaging was conducted at ambient pressure and room temperature at constant current mode with a Veeco Multimode III (Bruker) connected with a STM head (tip: mechanically cut $\mathrm{Pt} / \mathrm{Ir}$ wire, $4: 1, \theta=0.25 \mathrm{~mm}$, Goodfellow) and a 1 $\mu \mathrm{m}$ piezoelectric scanner (A-Piezo, Veeco). The substrates (HOPG, Momentive Performance) were glued on (silver conductive paint, Aldrich) a metal disk (Ted Pella) that magnetically attached to the STM base. In our instrumental configuration, the sample is grounded. The experiments were performed at the solid/liquid interface between the HOPG and a drop $(4 \mu \mathrm{L})$ of TCB solution of the molecule under investigation. The photocontrolled assembly was achieved by in situ irradiation of the solution on the HOPG substrate with optical fibercoupled LED light source (ThorLabs, $365 \mathrm{~nm}, P_{\mathrm{d}}=2 \mathrm{~mW} \mathrm{~cm}^{-2}$ ) by placing the collimator lens at a distance of $5 \mathrm{~cm}$ for $3 \mathrm{~min}$. The raw STM data were processed by SPIP (Image Metrology). Drift of every image was calibrated by the images of the HOPG crystal lattice in situ $\left(V_{\mathrm{T}}=60 \mathrm{mV}, I_{\mathrm{T}}=20 \mathrm{pA}\right)$. The unit cell constants were estimated from the average of multiple images ( $p$ AHA ori: 75, pAHA irr: 18, pAHA irr2: 6, oAHA ori: 13, oAHA ori2: 20, oAHA irr: 26).

MM/MD Simulations. The methodology employed in the MM/ MD simulation is the same as the one used in our previous work for multiazobenzene compounds. ${ }^{37}$ The details about the description of the modifed Dreiding force field ${ }^{52}$ used to reproduce the geometries of the multiazobenzene groups adsorbed on graphite are given in the previously mentioned work. All the $\mathrm{MM} / \mathrm{MD}$ simulations were performed using Materials Studio 7.0 package $^{53}$ Details of the simulation methods and description can be found in the Supporting Information.

\section{ASSOCIATED CONTENT}

\section{Supporting Information}

The Supporting Information is available free of charge on the ACS Publications website at DOI: 10.1021/acs.chemmater.9b01535.

${ }^{1} \mathrm{H}$ NMR and ${ }^{13} \mathrm{C}$ NMR characterization of the materials, UV-vis absorption spectra, additional STM images and lattice constants, and summary of $\mathrm{MM} / \mathrm{MD}$ figures and lattice constants (PDF)

\section{AUTHOR INFORMATION}

\section{Corresponding Authors}

*(A.N.) E-mail: narita@mpip-mainz.mpg.de.

*(P.S.) E-mail: samori@unistra.fr.

*(K.M.) E-mail: muellen@mpip-mainz.mpg.de. ORCID

Michal Valášek: 0000-0001-9382-6327

Marcel Mayor: 0000-0002-8094-7813

Jérôme Cornil: 0000-0002-5479-4227

Akimitsu Narita: 0000-0002-3625-522X

Paolo Samorì: 0000-0001-6256-8281

Klaus Müllen: 0000-0001-6630-8786

\section{Notes}

The authors declare no competing financial interest.

\section{ACKNOWLEDGMENTS}

This work was supported by the Max Planck Society, the EC through the Marie Sklodowska-Curie ITN project iSwitch (GA-642196). The activity in Strasbourg was also supported by the Agence Nationale de la Recherche through the LabEx project Chemistry of Complex Systems (ANR-10-LABX0026_CSC) and the International Center for Frontier Research in Chemistry (icFRC). We thank Steven D. Aird for editing the manuscript.

\section{REFERENCES}

(1) Narita, A.; Wang, X. Y.; Feng, X.; Müllen, K. New Advances in Nanographene Chemistry. Chem. Soc. Rev. 2015, 44 (18), 66166643.

(2) Wang, X.-Y.; Narita, A.; Müllen, K. Precision Synthesis versus Bulk-Scale Fabrication of Graphenes. Nat. Rev. Chem. 2018, 2 (1), 0100.

(3) Narita, A.; Chen, Z.; Chen, Q.; Müllen, K. Solution and OnSurface Synthesis of Structurally Defined Graphene Nanoribbons as a New Family of Semiconductors. Chem. Sci. 2019, 10 (4), 964-975.

(4) Chen, Q.; Brambilla, L.; Daukiya, L.; Mali, K. S.; De Feyter, S.; Tommasini, M.; Müllen, K.; Narita, A. Synthesis of Triply Fused Porphyrin-Nanographene Conjugates. Angew. Chem., Int. Ed. 2018, 57 (35), 11233-11237.

(5) Yen, H. J.; Tsai, H.; Zhou, M.; Holby, E. F.; Choudhury, S.; Chen, A.; Adamska, L.; Tretiak, S.; Sanchez, T.; Iyer, S.; et al. Structurally Defined 3D Nanographene Assemblies via Bottom-Up Chemical Synthesis for Highly Efficient Lithium Storage. Adv. Mater. 2016, 28 (46), 10250-10256.

(6) Wu, J.; Pisula, W.; Müllen, K. Graphenes as Potential Material for Electronics. Chem. Rev. 2007, 107 (3), 718-747.

(7) Wang, Y.; Yin, Z.; Zhu, Y.; Gu, J.; Li, Y.; Wang, J. Hexapole [9]Helicene. Angew. Chem., Int. Ed. 2019, 58 (2), 587-591.

(8) Evans, P. J.; Ouyang, J.; Favereau, L.; Crassous, J.; Fernández, I.; Perles, J.; Martín, N. Synthesis of a Helical Bilayer Nanographene. Angew. Chem., Int. Ed. 2018, 57 (23), 6774-6779.

(9) Ziogos, O. G.; Konstantinopoulos, S.; Tsetseris, L.; Theodorou, D. N. Computational Studies of Nanographene Systems: Extended 
Discotics, Covalently Linked "Supermolecules," and Functionalized Supramolecular Assemblies. J. Phys. Chem. C 2018, 122 (32), 1871518731 .

(10) Feng, X.; Pisula, W.; Kudernac, T.; Wu, D.; Zhi, L.; De Feyter, S.; Müllen, K. Controlled Self-Assembly of C3-Symmetric Hexa-PeriHexabenzocoronenes with Alternating Hydrophilic and Hydrophobic Substituents in Solution, in the Bulk, and on a Surface. J. Am. Chem. Soc. 2009, 131 (12), 4439-4448.

(11) Zhou, Y.; Zhang, M. Y.; Gu, K. H.; Zhu, Y. F.; Fan, X. H.; Shen, Z. Facile Synthesis and Phase Behaviors of Monofunctionalized HexaPeri-Hexabenzocoronenes. Asian J. Org. Chem. 2015, 4 (8), 746-755.

(12) Wong, W. W. H.; Singh, T. B.; Vak, D.; Pisula, W.; Yan, C.; Feng, X.; Williams, E. L.; Chan, K. L.; Mao, Q.; Jones, D. J.; et al. Solution Processable Fluorenyl Hexa-Peri-Hexabenzocoronenes in Organic Field-Effect Transistors and Solar Cells. Adv. Funct. Mater. 2010, 20 (6), 927-928.

(13) Gu, K.; Zhang, M.; Zhou, Y.; Han, M.; Zhang, W.; Shen, Z.; Fan, X. Hierarchical Self-Assembly of Disc-Rod Shape Amphiphiles Having Hexa-Peri-Hexabenzocoronene and a Relatively Long Rod. Langmuir 2017, 33 (13), 3311-3316.

(14) Hu, Y.; Dössel, L. F.; Wang, X.-Y.; Mahesh, S.; Pisula, W.; De Feyter, S.; Feng, X.; Müllen, K.; Narita, A. Synthesis, Photophysical Characterization, and Self-Assembly of Hexa- Peri -Hexabenzocoronene/Benzothiadiazole Donor-Acceptor Structure. ChemPlusChem 2017, 82 (7), 1030-1033.

(15) Keerthi, A.; Hou, I. C. Y.; Marszalek, T.; Pisula, W.; Baumgarten, M.; Narita, A. Hexa-Peri-Hexabenzocoronene with Different Acceptor Units for Tuning Optoelectronic Properties. Chem. - Asian J. 2016, 11 (19), 2710-2714.

(16) Hinkel, F.; Cho, D.; Pisula, W.; Baumgarten, M.; Müllen, K. Alternating Donor-Acceptor Arrays from Hexa-Perihexabenzocoronene and Benzothiadiazole: Synthesis, Optical Properties, and SelfAssembly. Chem. - Eur. J. 2015, 21 (1), 86-90.

(17) Wu, S. H.; Chen, H. H. Self-Assembling Behavior of Binary Mixture of Hexa-Peri-Hexabenzocoronene Derivatives with Different Molecular Symmetry. Tetrahedron 2019, 75 (2), 220-229.

(18) Dumslaff, T.; Yang, B.; Maghsoumi, A.; Velpula, G.; Mali, K. S.; Castiglioni, C.; De Feyter, S.; Tommasini, M.; Narita, A.; Feng, X.; et al. Adding Four Extra K-Regions to Hexa-Peri-Hexabenzocoronene. J. Am. Chem. Soc. 2016, 138 (14), 4726-4729.

(19) Ai, M.; Groeper, S.; Zhuang, W.; Dou, X.; Feng, X.; Müllen, K.; Rabe, J. P. Optical Switching Studies of an Azobenzene Rigidly Linked to a Hexa-Peri-Hexabenzocoronene Derivative in Solution and at a Solid-Liquid Interface. Appl. Phys. A: Mater. Sci. Process. 2008, 93 (2), 277-283.

(20) Pisula, W.; Zorn, M.; Chang, J. Y.; Müllen, K.; Zentel, R. Liquid Crystalline Ordering and Charge Transport In Semiconducting Materials. Macromol. Rapid Commun. 2009, 30 (14), 1179-1202.

(21) Fleischmann, E. K.; Zentel, R. Liquid-Crystalline Ordering as a Concept in Materials Science: From Semiconductors to StimuliResponsive Devices. Angew. Chem., Int. Ed. 2013, 52 (34), 88108827.

(22) Oda, K.; Hiroto, S.; Shinokubo, H. NIR Mechanochromic Behaviours of a Tetracyanoethylene-Bridged Hexa-: Peri-Hexabenzocoronene Dimer and Trimer through Dissociation of C-C Bonds. J. Mater. Chem. C 2017, 5 (22), 5310-5315.

(23) Feringa, B. L.; Browne, W. R. Molecular Switches, 2nd.; Feringa, B. L., Browne, W. R., Eds.; Wiley-VCH Verlag GmbH \& Co. KGaA: Weinheim, Germany, 2011; Vol. 1.

(24) Lubbe, A. S.; Szymanski, W.; Feringa, B. L. Recent Developments in Reversible Photoregulation of Oligonucleotide Structure and Function. Chem. Soc. Rev. 2017, 46 (4), 1052-1079.

(25) Balzani, V.; Credi, A.; Venturi, M. Molecular Devices and Machines: Concepts and Perspectives for the Nanoworld; 2nd ed.; WileyVCH Verlag GmbH \& Co. KGaA: Weinheim, Germany, 2008; Vol. 2.

(26) Zhang, X.; Hou, L.; Samorì, P. Coupling Carbon Nanomaterials with Photochromic Molecules for the Generation of Optically Responsive Materials. Nat. Commun. 2016, 7, 11118.
(27) Barrett, C. J.; Mamiya, J. I.; Yager, K. G.; Ikeda, T. PhotoMechanical Effects in Azobenzene-Containing Soft Materials. Soft Matter 2007, 3 (10), 1249-1261.

(28) Naumov, P.; Chizhik, S.; Panda, M. K.; Nath, N. K.; Boldyreva, E. Mechanically Responsive Molecular Crystals. Chem. Rev. 2015, 115 (22), 12440-12490.

(29) Bisoyi, H. K.; Li, Q. Light-Driven Liquid Crystalline Materials: From Photo-Induced Phase Transitions and Property Modulations to Applications. Chem. Rev. 2016, 116 (24), 15089-15166.

(30) Yagai, S.; Kitamura, A. Recent Advances in Photoresponsive Supramolecular Self-Assemblies. Chem. Soc. Rev. 2008, 37 (8), 15201529.

(31) Orgiu, E.; Samorì, P. 25th Anniversary Article: Organic Electronics Marries Photochromism: Generation of Multifunctional Interfaces, Materials, and Devices. Adv. Mater. 2014, 26 (12), 18271844.

(32) Scheil, K.; Gopakumar, T. G.; Bahrenburg, J.; Temps, F.; Maurer, R. J.; Reuter, K.; Berndt, R. Switching of an AzobenzeneTripod Molecule on $\operatorname{Ag}(111)$. J. Phys. Chem. Lett. 2016, 7 (11), 2080-2084.

(33) Jaekel, S.; Richter, A.; Lindner, R.; Bechstein, R.; Nacci, C.; Hecht, S.; Kühnle, A.; Grill, L. Reversible and Efficient Light-Induced Molecular Switching on an Insulator Surface. ACS Nano 2018, 12 (2), $1821-1828$

(34) Mielke, J.; Selvanathan, S.; Peters, M.; Schwarz, J.; Hecht, S.; Grill, L. Molecules with Multiple Switching Units on a $\mathrm{Au}(111)$ Surface: Self-Organization and Single-Molecule Manipulation. J. Phys.: Condens. Matter 2012, 24 (39), 394013.

(35) Tegeder, P. Optically and Thermally Induced Molecular Switching Processes at Metal Surfaces. J. Phys.: Condens. Matter 2012, 24 (39), 394001.

(36) Nacci, C.; Baroncini, M.; Credi, A.; Grill, L. Reversible Photoswitching and Isomer-Dependent Diffusion of Single Azobenzene Tetramers on a Metal Surface. Angew. Chem., Int. Ed. 2018, 57 (46), 15034-15039.

(37) Galanti, A.; Diez-Cabanes, V.; Santoro, J.; Valášek, M.; Minoia, A.; Mayor, M.; Cornil, J.; Samorì, P. Electronic Decoupling in C3Symmetrical Light-Responsive Tris(Azobenzene) Scaffolds: SelfAssembly and Multiphotochromism. J. Am. Chem. Soc. 2018, 140 (47), 16062-16070.

(38) Frath, D.; Yokoyama, S.; Hirose, T.; Matsuda, K. Photoresponsive Supramolecular Self-Assemblies at the Liquid/Solid Interface. J. Photochem. Photobiol., C 2018, 34, 29-40.

(39) Garah, M. El; Borré, E.; Ciesielski, A.; Dianat, A.; Gutierrez, R.; Cuniberti, G.; Bellemin-Laponnaz, S.; Mauro, M.; Samorì, P. LightInduced Contraction/Expansion of 1D Photoswitchable Metallopolymer Monitored at the Solid-Liquid Interface. Small 2017, 13 (40), 1701790.

(40) Zeitouny, J.; Aurisicchio, C.; Bonifazi, D.; De Zorzi, R.; Geremia, S.; Bonini, M.; Palma, C. A.; Samorì, P.; Listorti, A.; Belbakra, A.; et al. Photoinduced Structural Modifications in Multicomponent Architectures Containing Azobenzene Moieties as Photoswitchable Core. J. Mater. Chem. 2009, 19 (27), 4715-4724.

(41) Liu, Y.; Mu, L.; Liu, B.; Kong, J. Controlled Switchable Surface. Chem. - Eur. J. 2005, 11 (9), 2622-2631.

(42) Tahara, K.; Inukai, K.; Adisoejoso, J.; Yamaga, H.; Balandina, T.; Blunt, M. O.; De Feyter, S.; Tobe, Y. Tailoring Surface-Confined Nanopores with Photoresponsive Groups. Angew. Chem., Int. Ed. 2013, 52 (32), 8373-8376.

(43) Ito, S.; Wehmeier, M.; Brand, J. D.; Kübel, C.; Epsch, R.; Rabe, J. P.; Müllen, K. Synthesis and Self-Assembly of Functionalized HexaPeri-Hexabenzocoronenes. Chem. - Eur. J. 2000, 6 (23), 4327-4342.

(44) Harvey, J. H.; Butler, B. K.; Trauner, D. Functionalized Azobenzenes through Cross-Coupling with Organotrifluoroborates. Tetrahedron Lett. 2007, 48 (9), 1661-1664.

(45) Bléger, D.; Ciesielski, A.; Samorì, P.; Hecht, S. Photoswitching Vertically Oriented Azobenzene Self-Assembled Monolayers at the Solid-Liquid Interface. Chem. - Eur. J. 2010, 16 (48), 14256-14260. 
(46) Samorí, P.; Fechtenkötter, A.; Jäckel, F.; Böhme, T.; Müllen, K.; Rabe, J. P. Supramolecular Staircase via Self-Assembly of Disklike Molecules at the Solid-Liquid Interface. J. Am. Chem. Soc. 2001, 123 (46), 11462-11467.

(47) Piot, L.; Marchenko, A.; Wu, J.; Müllen, K.; Fichou, D. Structural Evolution of Hexa-Peri-Hexabenzocoronene Adlayers in Heteroepitaxy on n-Pentacontane Template Monolayers. J. Am. Chem. Soc. 2005, 127 (46), 16245-16250.

(48) Müllen, K.; Fichou, D.; Silly, F.; Marie, C.; Tortech, L. Tuning the Packing Density of 2D Supramolecular Self-Assemblies at the Solid-Liquid Interface Using Variable Temperature. ACS Nano 2010, 4 (3), 1288-1292.

(49) Cojal González, J. D.; Iyoda, M.; Rabe, J. P. Reversible Photoisomerization of Monolayers of $\pi$-Expanded Oligothiophene Macrocycles at Solid-Liquid Interfaces. Angew. Chem., Int. Ed. 2018, 57 (52), 17038-17042.

(50) Tahara, K.; Inukai, K.; Adisoejoso, J.; Yamaga, H.; Balandina, T.; Blunt, M. O.; De Feyter, S.; Tobe, Y. Tailoring Surface-Confined Nanopores with Photoresponsive Groups. Angew. Chem., Int. Ed. 2013, 52 (32), 8373-8376.

(51) Weippert, J.; Hauns, J.; Bachmann, J.; Böttcher, A.; Yao, X.; Yang, B.; Narita, A.; Müllen, K.; Kappes, M. M. A TPD-Based Determination of the Graphite Interlayer Cohesion Energy. J. Chem. Phys. 2018, 149 (19), 194701.

(52) Mayo, S. L.; Olafson, B. D.; Goddard, W. A. DREIDING: A Generic Force Field for Molecular Simulations. J. Phys. Chem. 1990, 94 (26), 8897-8909.

(53) MS Modeling, V7; Accelrys Software Inc.: San Diego, CA, 2015. 